\title{
The endoscopic endonasal eustachian tube anterolateral mobilization strategy: minimizing the cost of the extreme-medial approach
}

\author{
Mohamed A. Labib, MDCM, ${ }^{1}$ Evgenii Belykh, MD, PhD, ${ }^{1}$ Claudio Cavallo, MD, ${ }^{1}$ \\ Xiaochun Zhao, MD, ${ }^{1}$ Daniel M. Prevedello, MD, ${ }^{2}$ Ricardo L. Carrau, MD, ${ }^{3}$ Andrew S. Little, MD, ${ }^{1}$ \\ Mauro A. T. Ferreira, MD, ${ }^{4}$ Mark C. Preul, MD, ${ }^{1}$ A. Samy Youssef, MD, PhD, ${ }^{5}$ and Peter Nakaji, MD ${ }^{1}$ \\ 1'Department of Neurosurgery, Barrow Neurological Institute, St. Joseph's Hospital and Medical Center, Phoenix, Arizona; \\ Departments of ${ }^{2}$ Neurosurgery and ${ }^{3}$ Otolaryngology-Head and Neck Surgery, Wexner Medical Center at The Ohio State \\ University, Columbus, Ohio; ' Department of Anatomy and Radiology, University Hospital, Federal University of Minas Gerais, \\ Belo Horizonte, Brazil; and ${ }^{5}$ Department of Neurosurgery, University of Colorado Medical Center, Aurora, Colorado
}

OBJECTIVE The ventral jugular foramen and the infrapetrous region are difficult to access through conventional lateral and posterolateral approaches. Endoscopic endonasal approaches to this region are obstructed by the eustachian tube (ET). This study presents a novel strategy for mobilizing the ET while preserving its integrity. Qualitative and quantitative comparisons with previous ET management paradigms are also presented.

METHODS Ten dry skulls were analyzed. Four ET management strategies were sequentially performed on a total of 6 sides of cadaveric head specimens. Four measurement groups were generated: in group A, the ET was intact and not mobilized; in group B, the ET was mobilized inferolaterally; in group C, the ET underwent anterolateral mobilization; and in group D, the ET was resected. ET range of mobilization, surgical exposure area, and surgical freedom were measured and compared among the groups.

RESULTS Wide exposure of the infrapetrous region and jugular foramen was achieved by removing the pterygoid process, unroofing the cartilaginous ET up to the level of the posterior aspect of the foramen ovale, and detaching the ET from the skull base and soft palate. Anterolateral mobilization of the ET facilitated significantly more retraction (a 126\% increase) of the ET than inferolateral mobilization (mean \pm SD: $20.8 \pm 11.2 \mathrm{~mm}$ vs $9.2 \pm 3.6 \mathrm{~mm}$ [p $=0.02]$ ). Compared with group $A$, groups $C$ and $D$ had enhanced surgical exposure $\left(142.5 \%\left[1176.9 \pm 935.7 \mathrm{~mm}^{2}\right]\right.$ and $155.9 \%[1242.0 \pm$ $1096.2 \mathrm{~mm}^{2}$ ], respectively, vs $485.4 \pm 377.6 \mathrm{~mm}^{2}$ for group $\mathrm{A}$ [both $\mathrm{p}=0.02$ ]). Furthermore, group $\mathrm{C}$ had a significantly larger surgical exposure area than group $B(p=0.02)$. No statistically significant difference was found between the area of exposure obtained by ET removal and anterolateral mobilization. Anterolateral mobilization of the ET resulted in a $39.5 \%$ increase in surgical freedom toward the exocranial jugular foramen compared with that obtained through inferolateral mobilization of the ET $\left(67.2^{\circ} \pm 20.5^{\circ}\right.$ vs $\left.48.1^{\circ} \pm 6.7^{\circ}[p=0.047]\right)$ and a $65.4 \%$ increase compared with that afforded by an intact ET position $\left(67.2^{\circ} \pm 20.5^{\circ}\right.$ vs $\left.40.6^{\circ} \pm 14.3^{\circ}[p=0.03]\right)$.

CONCLUSIONS Anterolateral mobilization of the ET provides excellent access to the ventral jugular foramen and infrapetrous region. The surgical exposure obtained is superior to that achieved with other ET management strategies and is comparable to that obtained by ET resection.

https://thejns.org/doi/abs/10.3171/2019.12.JNS192285

KEYWORDS clivus; eustachian tube; extreme-medial approach; hypoglossal canal; jugular foramen; petroclival fissure; skull base

$\mathrm{T}$ RADITIONAL access to the jugular foramen and adjacent area is afforded via postauricular transtemporal, retrosigmoid, extreme-lateral transcondylar, and preauricular subtemporal-infratemporal approaches or by combinations thereof. ${ }^{2,4-7,12,14}$ Rhoton $^{13}$ eloquently summarized the inherent challenges associated with these approaches, stating, "The difficulties in exposing this foramen are created by its deep location and its proximity to critical anatomic structures, such as the carotid artery anteriorly, the facial nerve laterally, the hypoglossal nerve

ABBREVIATIONS CN = cranial nerve; $E T$ = eustachian tube; ICA = internal carotid artery.

SUBMITTED August 20, 2019. ACCEPTED December 16, 2019.

INCLUDE WHEN CITING Published online March 13, 2020; DOI: 10.3171/2019.12.JNS192285. 
medially, and the vertebral artery inferiorly, all of which block access to the foramen and require careful management."

In the past decade, endoscopic endonasal approaches to the jugular foramen and infrapetrous region have evolved as alternatives for resecting select lesions that originate at the petroclival fissure and displace the lower cranial nerves (CNs) dorsally or laterally. ${ }^{10,11,16}$ Although some of the anatomical constraints described previously by Rhoton may not compromise the endoscopic approaches, these approaches are significantly restricted by the anatomical position of the eustachian tube (ET). Two strategies to manage the ET have been complete resection, which risks chronic serous otitis and conductive hearing loss, and inferolateral mobilization, which does not provide full exposure of the jugular foramen.

We propose a novel strategy-anterolateral mobilization - to mobilize the ET laterally and create direct access to the jugular foramen, hypoglossal orifice, and parapharyngeal internal carotid artery (ICA), while obviating the need to use an angled endoscope. Detailed quantitative comparisons of this new strategy with inferolateral mobilization or complete resection of the ET were performed in cadaveric specimens.

\section{Methods}

The study was conducted at the surgical neuroanatomy laboratory of Barrow Neurological Institute (St. Joseph's Hospital and Medical Center). Osteological relationships were analyzed in 10 dry skulls. Six sides of silicone-injected cadaveric specimens were dissected. Endonasal dissections were performed using standard endoscopes (4$\mathrm{mm} \times 18-\mathrm{cm}$ Hopkins II; Karl Storz \& Co. KG) attached to a high-definition camera. An illustrative case where the anterolateral mobilization strategy would have been useful is included.

\section{Quantitative Analysis}

A navigation system (StealthStation; Medtronic) was used to perform measurements. Different ET management strategies were sequentially performed on each of the 6 sides of the cadaveric specimens. Four measurement groups were generated: in group A, the ET was not mobilized; in group B, the ET was mobilized inferolaterally; in group C, the ET underwent anterolateral mobilization; and in group D, the ET was resected. Figure 1 depicts the relevant areas and angles that were measured.

The range of ET mobilization was defined as the distance between the center of the ET opening in its natural position and its opening after mobilization. The surgical area of exposure was defined as a surface of the polygon limited by points 1 through 5 (Fig. 1). Surgical freedom was assessed as a quantitative value of the narrowness of the corridor created by the approach. For this assessment, angles of exposure within the boundaries of the approach toward predetermined anatomical landmarks were used as surrogates to evaluate surgical freedom. Angles toward the exocranial orifice of two anatomical landmarks (jugular foramen and hypoglossal canal) were measured using the coordinates of each landmark as their vertices. The constant position of one of the rays forming the angles was defined as a ray originating at the anatomical landmark (jugular foramen or hypoglossal canal) and pointing to the parapharyngeal tubercle (i.e., midline). A second ray originated at the anatomical landmark (jugular foramen or hypoglossal canal) and pointed to the center of the orifice of the ET in each of the 3 evaluated positions, thus reflecting the degree of surgical freedom with each paradigm.

\section{Results \\ Relevant Osteological Landmarks}

Several anatomical relationships are of interest (Fig. 2). First, the foramen ovale is located posterolateral to the posterior aspect of the lateral pterygoid plate. The foramen has two lips that converge at its anterior and posterior edges. We labeled the lateral and medial lips the "lateral ovale process" and the "medial ovale process," respectively. The anterior third of the medial ovale process, which is relevant to the anterolateral mobilization technique, veers anteriorly toward the posterior edge of the lateral pterygoid plate.

Second, the medial pterygoid plate diverges at the level of the posterior opening of the vidian canal into two tubercles. The medial tubercle is the pterygoid tubercle and is inferomedial to the vidian canal. The lateral tubercle is a posterior continuation of the scaphoid fossa, and it merges with the medial ovale process. Because of the intimate relationship of the lateral tubercle to the scaphoid fossa, we termed it the "scaphoid tubercle" (Fig. 2). In the 10 dry skulls (20 sides), the mean ( \pm SD) widths of the scaphoid and pterygoid tubercles were $6.43 \pm 1.37 \mathrm{~mm}$ and $5.70 \pm$ $1.14 \mathrm{~mm}$, respectively. The mean length of the pterygoid tubercle was $3.38 \pm 1.36 \mathrm{~mm}$ (Table 1).

Third, the exocranial caudal aspect of the foramen lacerum is triangular in shape and has 3 borders: the anterior border is formed by the pterygoid process of the sphenoid bone, the medial border by the clival segment of the occipital bone, and the posterior border by the petrous segment of the temporal bone. At the anterior margin of foramen lacerum is the pterygoid tubercle; this tubercle is slightly inferomedial to the posterior orifice of the vidian canal. Staying in the axial plane of the pterygoid tubercle while dividing the fibrocartilage attaching the ET to the foramen lacerum avoids violating the overlying ICA. We therefore consider the pterygoid tubercle to be an important landmark for defining the superior limit of any incision intended to detach the ET from the fibrocartilaginous tissue of the foramen lacerum.

Finally, the sphenopetrosal fissure where the fibrocartilaginous attachment of the ET inserts along the cranial base extends medially to laterally in the coronal plane and anteriorly to posteriorly in the sagittal plane. Thus, the line of the incision for detaching the ET from the skull base should follow the exact trajectory of the sphenopetrosal fissure.

\section{Surgical Anatomy of the ET}

The cartilaginous portion of the ET is divided into 3 segments: posterolateral, middle, and anteromedial. The posterolateral segment is attached superiorly to the sulcus 

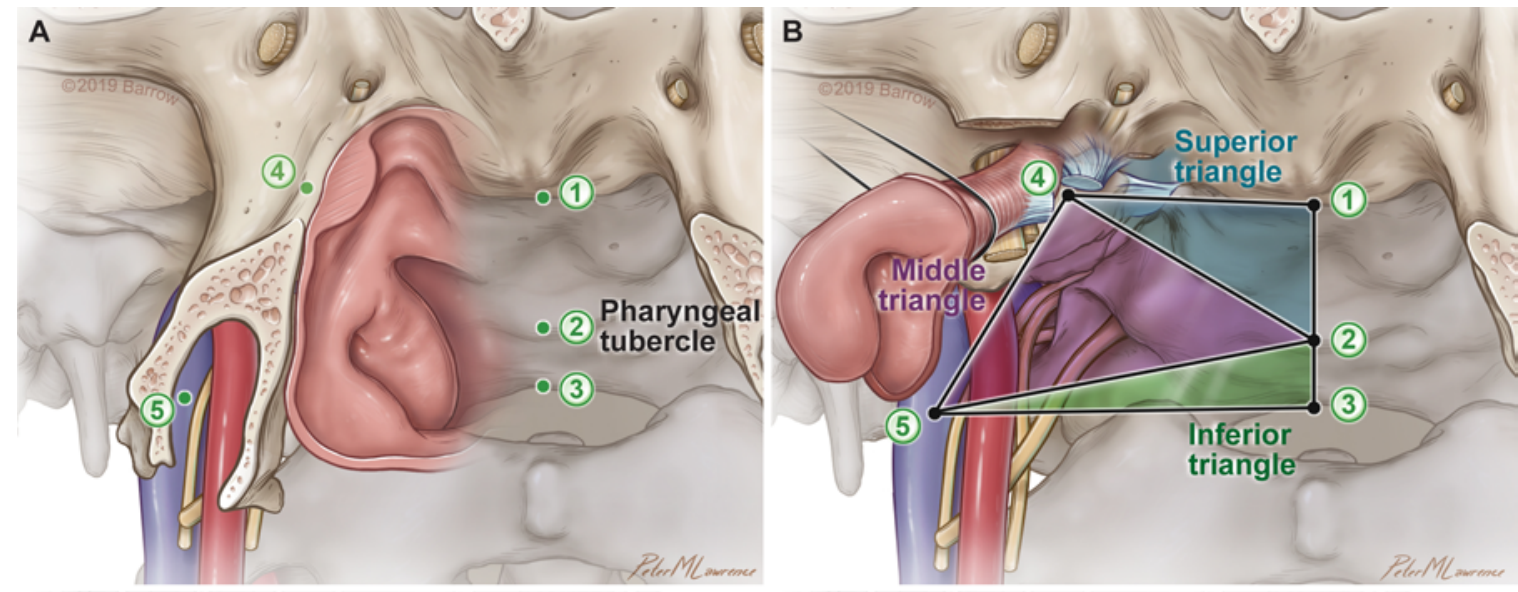

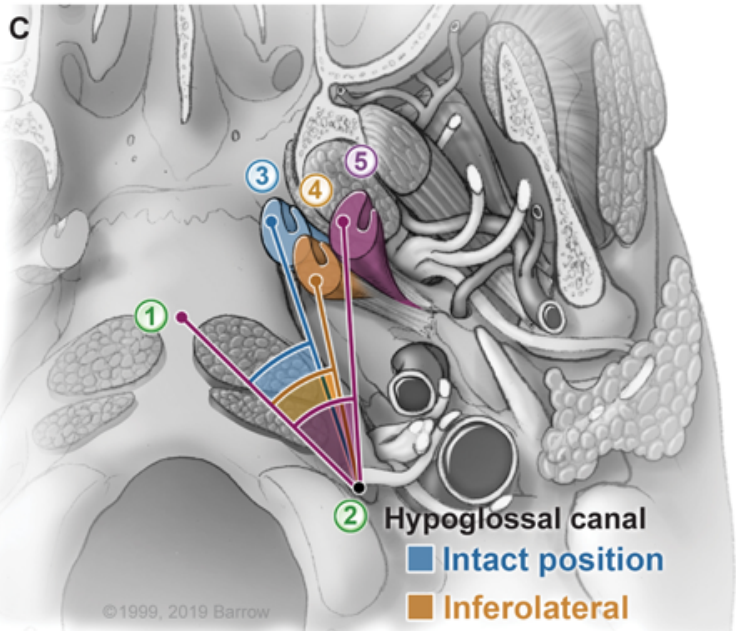

Anterolateral

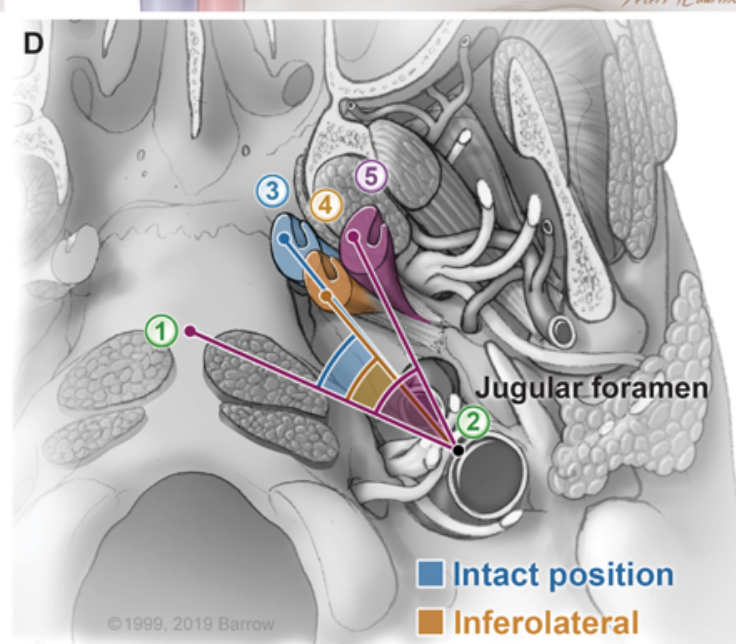

Anterolateral

FIG. 1. Anatomical landmarks used for approach quantification. A: Positions of the anatomical landmarks for quantification of the surgical exposure area: (1) midline, clivus, at the junction with the posterior aspect of the vomer; (2) midline, clivus, at the pharyngeal tubercle; (3) midline, clivus, at the anterior lip of the foramen magnum; (4) the most superolateral point of the exposure; and (5) the most inferolateral point of the exposure. B: The sum of the surface areas formed by the triangles was used to calculate the exposure area. Numbers in panel B represent the same anatomy as those in panel A. C: Positions of the anatomical landmarks used for quantifying the angles of exposure of the hypoglossal canal with each of the ET management strategies: (1) midline, clivus, at the pharyngeal tubercle; (2) exocranial orifice of the hypoglossal canal; (3) intact ET position without mobilization; (4) ET position with inferolateral mobilization; and (5) ET position with anterolateral mobilization. D: Positions of the anatomical landmarks used for quantifying the angles of exposure of the jugular foramen with each of the ET management strategies: (1) midline, clivus, at the pharyngeal tubercle; (2) exocranial orifice of the ventral aspect of the jugular foramen; (3) ET position without mobilization; (4) ET position with inferolateral mobilization; and (5) ET position with anterolateral mobilization. Copyright Barrow Neurological Institute, Phoenix, Arizona. Published with permission.

tubae along the sphenopetrosal fissure. The middle segment courses anterolaterally to the foramen lacerum, below the scaphoid and pterygoid tubercles. The anteromedial segment is inferior to the scaphoid fossa laterally and the vidian canal medially, and it is medial to the medial pterygoid plate (Fig. 2).

\section{Anterolateral Mobilization of the ET: Technical Nuances}

The steps for anterolateral mobilization of the ET are outlined below (Figs. 3 and 4) and are illustrated in Video 1.

VIDEO 1. Anterolateral mobilization of the eustachian tube. Copyright Barrow Neurological Institute, Phoenix, Arizona.

Published with permission. Click here to view.

\section{Step 1: Extended Transsphenoidal Approach}

The extended transsphenoidal approach involving an ipsilateral middle turbinectomy, anterior and posterior ethmoidectomies, bilateral sphenoidotomies, and a posterior septectomy has been detailed elsewhere. ${ }^{8,9}$

\section{Step 2: Exposure of Pterygopalatine Fossa and Infratemporal Fossa}

A middle and inferior turbinectomy, uncinectomy, and removal of the medial wall of the maxilla are performed to expose the entire posterior wall of the antrum. The sphenopalatine foramen is identified posterior to the upper third of the antrum wall. As much of the inferior aspect of the lateral nasal wall as possible should be removed to allow the complete reduction of the inferior and lateral 

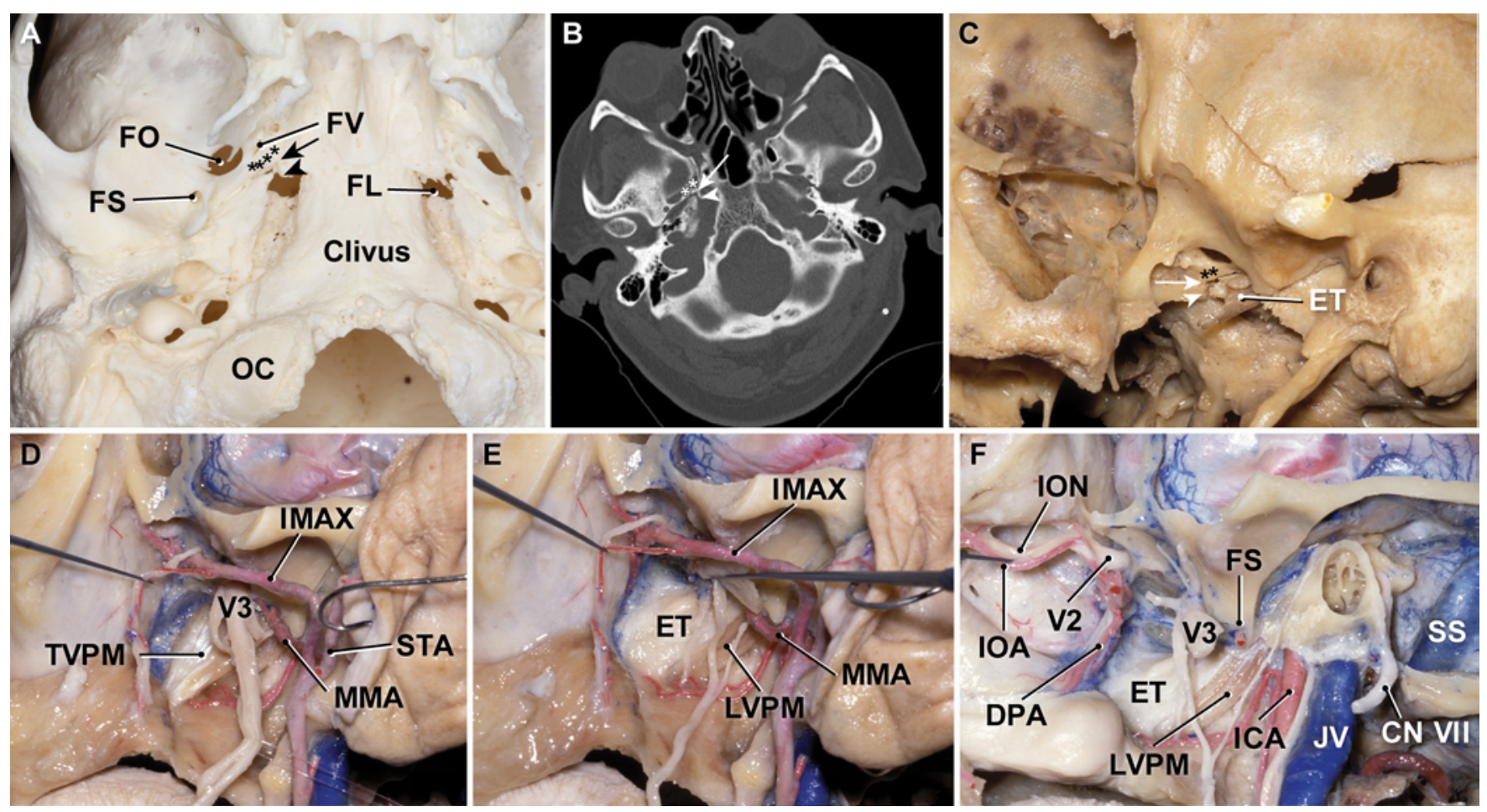

FIG. 2. The ventral skull base. A: Inferior view of a dry skull showing the relevant landmarks. B: Axial, thin-slice CT scan showing the inferior view of relevant landmarks. The vidian canal is at the junction of the body and pterygoid process of the sphenoid bone. The vidian canal has an anterior opening (not visible from this view) inferomedial to the foramen rotundum. The posterior opening of the vidian canal (arrow) is slightly more lateral to the anterior opening and is located superior to the posterior edge of the medial pterygoid plate. Two osseous projections are located inferior to the vidian canal and at the level of its posterior opening. The medial projection is the pterygoid tubercle (arrowhead), which marks the anterolateral aspect of the foramen lacerum. The lateral osseous protrusion borders the sphenopetrosal fissure and is a continuation of the borders of the scaphoid fossa; hence, we termed it the scaphoid tubercle (asterisks). Both the pterygoid and scaphoid tubercles are important landmarks for the ET anterolateral mobilization technique (see below). The foramen ovale is positioned posterolaterally to the lateral pterygoid process. The lateral and medial lips of the foramen ovale are termed the lateral ovale process and the medial ovale process, respectively. The medial ovale process is divided into thirds. The posterior two-thirds border the sphenopetrosal fissure, whereas the anterior one-third veers anteriorly toward the posterior edge of the lateral pterygoid plate. C: Lateral view of the left side of a dry skull after removing the zygomatic arch and the posterior wall of the maxillary sinus to expose the pterygoid process. The posterior opening of the vidian canal (arrow), the pterygoid tubercle (arrowhead), and the scaphoid tubercles (asterisks) are identified. The ET is identified medial to the foramen ovale. D: Cadaveric dissection of the left infratemporal and pterygopalatine fossae. The zygomatic arch is removed, the lateral pterygoid plate is partially removed, and the posterior wall of the maxillary sinus is removed while preserving its mucosa. The tensor veli palatini muscle (TVPM) is lateral to the ET. The mandibular nerve (V3) exits the foramen ovale posterior to the pterygoid process. The internal maxillary artery (IMAX) arises from the external carotid artery and courses in the infratemporal fossa anteriorly toward the pterygopalatine fossa, where it bifurcates into its terminal branches: the sphenopalatine artery and the descending palatine arteries. E: The TVPM has been divided, and V3 has been retracted to expose the cartilaginous part of the ET and the levator veli palatini muscle (LVPM). F: A close-up view after dividing V3 and the IMAX. The descending palatine artery (DPA) is a terminal branch of the IMAX, which courses inferiorly toward the soft and hard palates. The infraorbital artery and nerve course in the infraorbital groove, en route from the infraorbital fissure to the infraorbital foramen. The middle meningeal artery enters the foramen spinosum. $\mathrm{FL}=$ foramen lacerum; $\mathrm{FO}=$ foramen ovale; $\mathrm{FS}=$ foramen spinosum; $\mathrm{FV}$ = foramen of Vesalius; IOA = infraorbital artery; ION = infraorbital nerve; JV = jugular vein; MMA = middle meningeal artery; OC = occipital condyle; SS = sigmoid sinus; STA = superficial temporal artery; V2 = maxillary nerve. Panels A and C-F: Copyright Barrow Neurological Institute, Phoenix, Arizona. Published with permission.

margins of the posterior wall of the sinus. The posterior wall of the antrum is then removed to expose the pterygopalatine fossa, extending the removal laterally to the inferior orbital fissure and identifying the transition from the pterygopalatine to the infratemporal fossae through the pterygomaxillary fissure. The sphenopalatine and posterior nasal arteries are coagulated and divided to expose the vidian foramen and its neurovascular bundle, which is also coagulated and divided to aid in the lateralization of the contents of the pterygopalatine fossa.

\section{Step 3: Exposure and Removal of Pterygoid Process}

Because the pterygoid process is positioned lateral to the anteromedial segment of the ET, a key step in the anterolateral mobilization of the ET is the complete removal of the pterygoid process. The pterygoid process is fully exposed by removing the overlying ascending process of the palatine bone and opening the greater palatine canal. The contents of the canal-the descending palatine vessels and the greater and lesser palatine nerves-are dissected. The greater palatine nerve and descending palatine artery can 
TABLE 1. Morphometric parameters of the scaphoid and pterygoid tubercles

\begin{tabular}{|c|c|c|c|}
\hline $\begin{array}{l}\text { Specimen No. } \\
\text { \& Side }\end{array}$ & $\begin{array}{l}\text { Scaphoid Tubercle Width } \\
\qquad(\mathrm{mm})\end{array}$ & $\begin{array}{l}\text { Pterygoid Tubercle Width } \\
\qquad(\mathrm{mm})\end{array}$ & $\begin{array}{l}\text { Pterygoid Tubercle Length } \\
\qquad(\mathrm{mm})\end{array}$ \\
\hline \multicolumn{4}{|l|}{1} \\
\hline Right & 7 & 5 & 3.5 \\
\hline Left & 5.5 & 6 & 2.5 \\
\hline \multicolumn{4}{|l|}{2} \\
\hline Right & 7.5 & 6 & 2 \\
\hline Left & 8 & 6 & 2.5 \\
\hline \multicolumn{4}{|l|}{3} \\
\hline Right & 4.5 & 6.5 & 2.5 \\
\hline Left & 5 & 6 & 2.5 \\
\hline \multicolumn{4}{|l|}{4} \\
\hline Right & 5 & 5 & 2.5 \\
\hline Left & 5 & 5 & 3 \\
\hline \multicolumn{4}{|l|}{5} \\
\hline Right & 8.5 & 6 & 2 \\
\hline Left & 6.5 & 5 & 2.5 \\
\hline \multicolumn{4}{|l|}{6} \\
\hline Right & 7 & 5 & 3 \\
\hline Left & 7 & 5.5 & 2 \\
\hline \multicolumn{4}{|l|}{7} \\
\hline Right & 5 & 9 & 5 \\
\hline Left & 7 & 7 & 5 \\
\hline \multicolumn{4}{|l|}{8} \\
\hline Right & 8 & 7 & 7 \\
\hline Left & 9 & 5 & 5 \\
\hline \multicolumn{4}{|l|}{9} \\
\hline Right & 6 & 4 & 3 \\
\hline Left & 7 & 4 & 3 \\
\hline \multicolumn{4}{|l|}{10} \\
\hline Right & 5 & 6 & 4 \\
\hline Left & 5 & 5 & 5 \\
\hline
\end{tabular}

be preserved and lateralized, whereas the lesser palatine nerve is divided to facilitate access to the pterygoid process. The soft tissue of the pterygopalatine fossa can be dissected off the pterygoid process in a subperiosteal fashion and mobilized laterally to expose the anterior face of the pterygoid process and the foramen rotundum. The dissection trajectory then shifts laterally to the lateral pterygoid plate. The lateral pterygoid muscle and accompanying soft tissue are dissected in a subperiosteal fashion off the plate so as to avoid bleeding from the pterygoid plexus. The posterior limit of this dissection is the foramen ovale. Similarly, the medial pterygoid plate is dissected off the ET and its attached muscles and soft tissue (Fig. 3A).

After the exposure is completed, the pterygoid process is drilled (Fig. 3B). Two imaginary lines of drilling are established. The rostral line begins medially at the floor of the anterior orifice of the vidian canal and ascends laterally under the floor of the foramen rotundum and up to the lateral aspect of the pterygoid process. The drilling progresses along this line, from anterior to posterior, up to the level of the foramen ovale. The caudal line is also horizontal in the same plane as the floor of the nasal cavity and progresses posteriorly. The drilled bone is then freed off the soft tissue (pterygoid muscles) subperiosteally.

\section{Step 4: Unroofing the Cartilaginous ET}

The attachment of the ET cartilage to the skull base is exposed by drilling the overlying bone. First, any remnant of the sphenoid floor medial to the level of the paraclival ICA is drilled flush with the clival recess. Second, the floor of the vidian canal and the pterygoid tubercle inferomedial to it are completely drilled down to the level of the fibrocartilaginous layer of the foramen lacerum (Fig. 3C). The impression of the pterygoid tubercle in the fibrocartilage is evident as a depression we term the "pterygoid depression" (Fig. 3D). Third, the drilling plane shifts laterally to where the scaphoid tubercle connecting the medial pterygoid plate anteromedial to the medial ovale process posterolaterally is drilled along the sphenopetrosal fissure. The drilling is carried further posteriorly until the medial 
Labib et al.

A
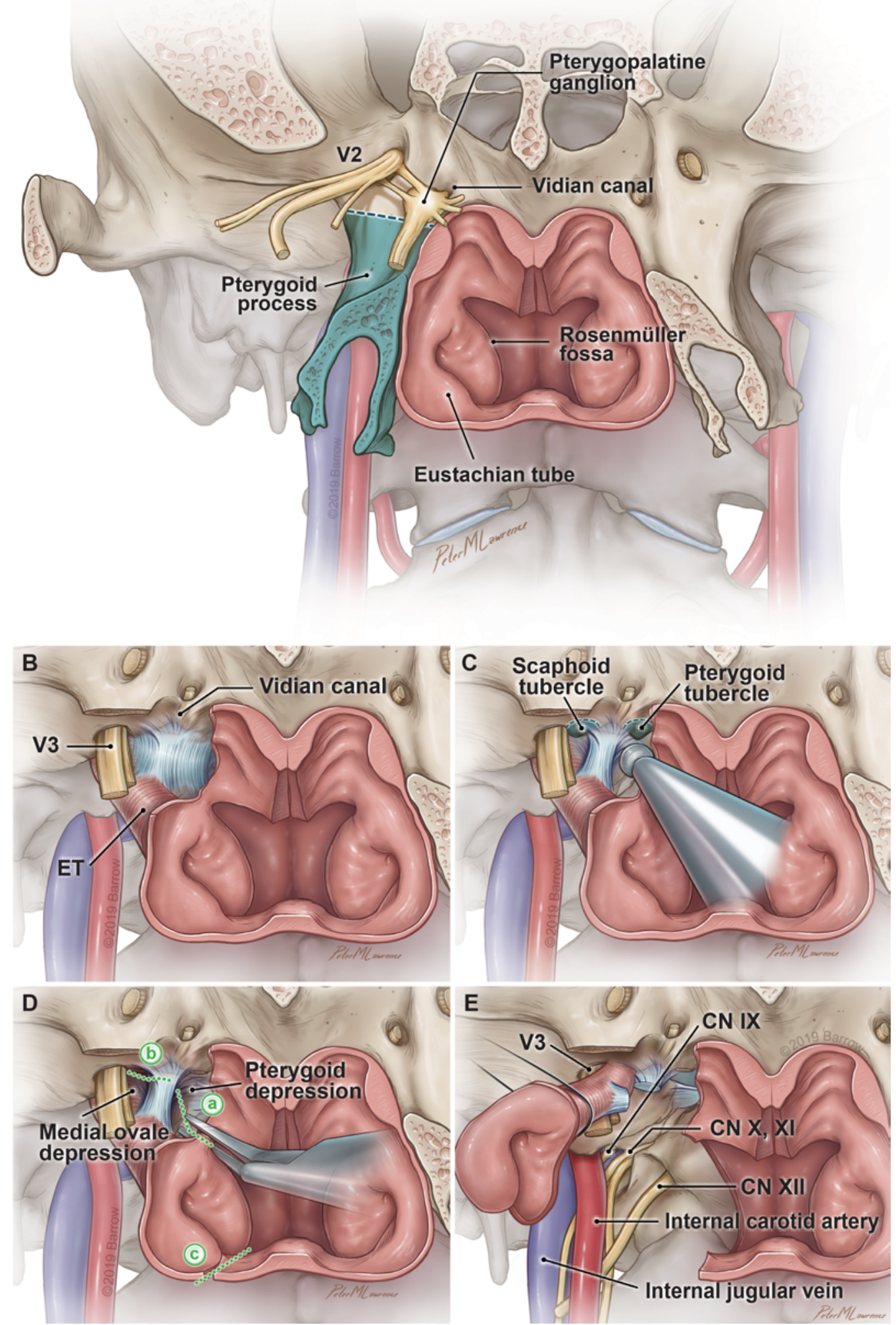

FIG. 3. Illustration of the ET anterolateral mobilization. A: Anterior view of a coronal section of the skull base. The vidian and maxillary nerves are identified. The segment of the pterygoid process (shaded in green) will be drilled to create sufficient space for mobilizing the ET anterolaterally. B: The pterygoid process is drilled, and the fibrocartilaginous tissue attaching the ET to the skull base is identified. C: The pterygoid and scaphoid tubercles (curved, dashed lines) are important landmarks inferomedial and inferolateral to the vidian canal, respectively. The pterygoid tubercle is drilled to expose the pterygoid depression. The scaphoid tubercle, which connects posteriorly to the medial ovale process, is drilled to expose the medial ovale depression. FIG. 3. (continued) $\rightarrow$ 
FIG. 3. D: The superior and inferior incisions (green dots). The first incision (a) to mobilize the ET starts at the upper aspect of the fossa of Rosenmüller and extends superiorly, posteriorly, and laterally up to the pterygoid depression, which is easily visualized. The second incision (b) extends from the pterygoid depression inferior to the vidian nerve and up to the medial ovale depression and ends immediately medial to the foramen ovale. The inferior incision (c) detaches the lower part of the ET from the soft palate. E: The view obtained after anterolateral ET mobilization shows where the lower cranial nerves exit the hypoglossal and jugular foramina. CN X = vagus nerve; CN XI = accessory nerve; CN XII = hypoglossal nerve; V2 = maxillary nerve; $\mathrm{V} 3$ = mandibular nerve. Copyright Barrow Neurological Institute, Phoenix, Arizona. Published with permission.

ovale process is completely removed. Although the lateralmost aspect of the cartilaginous ET extends posterolaterally on the sulcus tubae up to a level immediately medial to the sphenoid spine, we have found that drilling posterior to the medial ovale process is not necessary to accomplish ET mobilization.

\section{Step 5: Superior Detethering Incision}

The first incision (Fig. 3D [a]) to mobilize the ET starts at the upper aspect of the fossa of Rosenmüller and extends superiorly, posteriorly, and laterally up to the pterygoid depression, which is easily visualized. The second incision (Fig. 3D [b]) extends from the pterygoid depression inferior to the vidian nerve and up to the medial ovale depression. This incision ends at a level immediately medial to the foramen ovale. It is important to have adequately completed the drilling discussed above to facilitate these two incisions.

\section{Step 6: Inferior Nasopharyngeal Incision}

The inferior incision (Fig. 3D [c]) detaches the lower part of the ET from the soft palate. It starts anteriorly and extends posteriorly below the torus tubarius up to the inferior aspect of the fossa of Rosenmüller. The levator veli palatini and tensor veli palatini muscles remain attached to the ET.

\section{Extreme-Medial Approach With Anterolateral Mobilization of the ET}

After performing the anterolateral mobilization of the ET (Figs. 3 and 4), the nasopharyngeal mucosa and the underlying basipharyngeal fascia are incised and resected using monopolar cautery. The longus capitis and rectus capitis anterior muscles are then divided and reflected inferiorly. Several structures are identified, including the pharyngeal tubercle, supracondylar groove, atlantooccipital joint, and anterior lip of the foramen magnum. The clivus is then drilled from the level of the floor of the sphenoid sinus down to the foramen magnum. Laterally, the supracondylar groove is drilled to expose the cortical bone of the hypoglossal canal. Superior to this cortical bone is the jugular tubercle, and inferior to it is the occipital condyle. The cortical bone of the hypoglossal canal is drilled down to the dura. Similarly, the jugular tubercle is drilled and followed laterally toward the petrous compartment of the jugular foramen, behind which the lower cranial nerves are seen traversing the foramen (Fig. 4).

\section{Morphometric Analysis of ET Management Techniques}

The ET mobilization distance, surgical area of exposure, and surgical freedom were compared among all groups (Figs. 5 and 6).

\section{ET Mobilization Distance}

Anterolateral mobilization resulted in significantly more retraction of the ET (126\% increase in distance) in group $\mathrm{C}$ than inferolateral mobilization in group B (20.8 \pm $11.2 \mathrm{~mm}$ vs $9.2 \pm 3.6 \mathrm{~mm}[\mathrm{p}=0.02]$ ) (Fig. 6).

\section{Surgical Exposure Area}

Compared with the surgical exposure area in specimens with an intact ET (group A), surgical exposure was increased in specimens with inferolateral ET mobilization (group B), with anterolateral ET mobilization (group C), and with ET removal (group D) (Fig. 6). ET removal and anterolateral mobilization resulted in the largest increase in surgical exposure (mean increases $142.5 \%$ and $155.9 \%$, respectively) compared with the intact ET (1176.9 \pm 935.7 $\mathrm{mm}^{2}$ and $1242.0 \pm 1096.2 \mathrm{~mm}^{2}$, respectively, vs $485.4 \pm$ $377.6 \mathrm{~mm}^{2}$ [both $\left.\mathrm{p}=0.02\right]$ ). No statistically significant difference was found between the area of exposure obtained by ET removal and that obtained by anterolateral mobilization. Inferolateral ET mobilization provided a $73.6 \%$ increase in exposure over the intact ET $(843.0 \pm 643.9$ $\left.\mathrm{mm}^{2}[\mathrm{p}=0.02]\right)$. Anterolateral ET mobilization provided significantly greater surgical exposure than inferolateral mobilization ( $\mathrm{p}=0.02)$ (Fig. 6).

\section{Surgical Freedom}

Anterolateral ET mobilization resulted in significantly increased surgical freedom, with a $46.2 \%$ increase toward the external opening of the hypoglossal canal compared with inferolateral ET mobilization $\left(70.8^{\circ} \pm 33.0^{\circ}\right.$ vs $48.0^{\circ}$ $\pm 5.0^{\circ}[\mathrm{p}=0.03]$ ) and an $84.1 \%$ increase compared with the intact ET $\left(70.8^{\circ} \pm 33.0^{\circ}\right.$ vs $\left.38.5^{\circ} \pm 11.9^{\circ}[\mathrm{p}=0.03]\right)$ (Fig. 6). Anterolateral ET mobilization resulted in a 39.5\% increase in surgical freedom toward the exocranial opening of the jugular foramen compared with inferolateral ET mobilization $\left(67.2^{\circ} \pm 20.5^{\circ}\right.$ vs $\left.48.1^{\circ} \pm 6.7^{\circ}[\mathrm{p}=0.047]\right)$ and resulted in a $65.4 \%$ increase compared with the intact ET $\left(67.2^{\circ} \pm 20.5^{\circ}\right.$ vs $\left.40.6^{\circ} \pm 14.3^{\circ}[p=0.03]\right)$ (Fig. 6).

\section{Illustrative Case}

A 37-year-old woman presented with right abducens nerve palsy. Imaging showed a large lesion involving the right petroclival fissure and jugular foramen (Fig. 7). The patient underwent an endoscopic endonasal extrememedial approach with transection of the ET. The pathological findings were consistent with well-differentiated chondrosarcoma, WHO grade I. Gross-total resection was obtained, and the abducens nerve palsy subsequently resolved. The patient remains recurrence-free after 3 years. On the basis of our anatomical data, this is a case in which anterolateral mobilization of the ET would have yielded a similar exposure to that achieved with resection. 

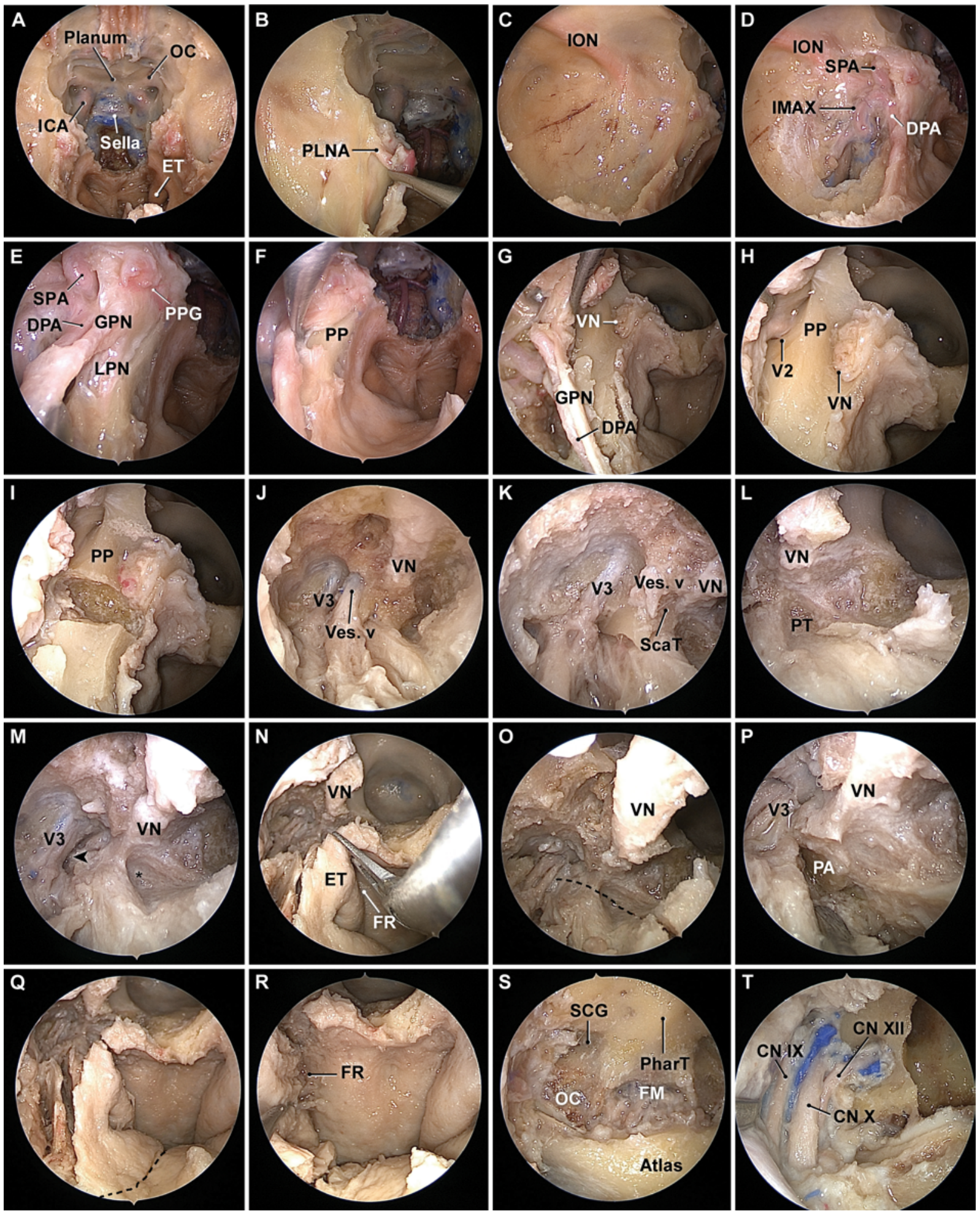

FIG. 4. Cadaveric illustrations of the ET mobilization. A: Panoramic view of the ventral skull base after completing anterior and posterior ethmoidectomies, posterior septectomy, and bilateral sphenoidotomies with complete reduction of the septae. B: A right medial maxillectomy was performed to expose the entire wall of the antrum. The posterior lateral nasal artery (PLNA), a branch of the sphenopalatine artery (SPA), was identified. C: The PLNA was divided. The infraorbital nerve (ION) travels in the infraorbital groove from the infraorbital fissure toward the infraorbital foramen. It is necessary to continue the exposure lateral to the inferior orbital fissure, as demarcated by the ION, because this exposure guarantees the transition from the pterygopalatine fossa to the infratemporal fossa through the pterygomaxillary fissure. D: The posterior wall of the maxillary sinus was removed, and the pterygopalatine and infratemporal fossae were exposed. The internal maxillary artery (IMAX) bifurcates into the SPA and descending palatine artery (DPA). The ION courses in the infraorbital groove. E and F: The DPA, greater palatine nerve (GPN), and lesser palatine nerve (LPN) are identified after opening the greater palatine canal. The GPN is preserved, while the LPN is divided. FIG. 4. (continued) $\rightarrow$ 
FIG. 4. G and H: The vidian nerve (VN) is divided off the pterygopalatine ganglion (PPG); this division allows for the lateralization of the ganglion and exposure of the pterygoid process (PP) without the need to divide the maxillary nerve. I: The PP was drilled below the level of the VN along an imaginary line beginning medially at the floor of the anterior orifice of the vidian canal and ascending laterally under the floor of the foramen rotundum up to the lateral aspect of the PP. J: The PP has been removed to expose the mandibular nerve posteriorly as it exits the foramen ovale and the vein of Vesalius as it exits through its foramen medial to the foramen ovale. K: The vein of Vesalius (Ves. v) has been divided, exposing the scaphoid tubercle (ScaT) lateral to the vidian canal. L: The pterygoid tubercle (PT) is exposed medial to the vidian canal. M: The PT has been drilled to expose the pterygoid depression (asterisk). The ScaT, which connects posteriorly to the medial ovale process, has been drilled to expose the medial ovale depression (arrowhead). N: The first incision to mobilize the ET starts at the upper aspect of the fossa of Rosenmüller (FR) and extends in a superior, posterior, and lateral direction up to the pterygoid depression. 0: The second incision (dashed line) extends from the pterygoid depression inferior to the VN and up to the medial ovale process depression. This incision ends at a level immediately medial to the foramen ovale. P: As the ET has been detached from the sulcus tubae and retracted laterally, the petrous bone medial to it becomes visible. Q: The inferior incision (dashed line) detaches the lower part of the ET from the soft palate; it starts anteriorly and extends posteriorly below the torus tubarius and extends up to the inferior aspect of the FR. R: At the completion of the ET anterolateral mobilization, the FR is open. S: The nasopharyngeal mucosa and the underlying basipharyngeal fascia and muscles are incised and resected to expose the clivus and the anterior arch of the atlas. Several landmarks can be identified, including the pharyngeal tubercle (PharT), the supracondylar groove (SCG), the atlantooccipital joint, and the anterior lip of the foramen magnum (FM). T: The supracondylar groove was followed laterally and then drilled to expose the cortical bone of the hypoglossal canal. The lower cranial nerves exit the jugular foramen. OC = occipital condyle; $P A=$ petrous apex. Copyright Barrow Neurological Institute, Phoenix, Arizona. Published with permission.

\section{Discussion}

\section{Jugular Foramen: Ultimate Challenge for Open and Endoscopic Approaches}

The jugular foramen is the second-most lateral major skull base foramen, with the horizontal distance from the midline to its lateral aspect at least double the distance from the jugular foramen to the mastoid tip. Nevertheless, 3 considerations may justify the feasibility of an endoscopic endonasal approach to the jugular foramen in select cases. First, the stylomastoid foramen is immediately lateral to the jugular foramen. Any direct lateral approach must expose, or even transpose, the mastoid segment of the facial nerve (CN VII) to gain full access to the lateral aspect of the jugular foramen. Such manipulation of the facial nerve may cause transient or even permanent facial palsy. Second, the neural part of the jugular foramen, through which the lower cranial nerves course on their way to the poststyloid compartment of the parapharyngeal space, is interposed between the posterolateral sigmoid compartment (receiving the sigmoid sinus) and the anterolateral petrous compartment (receiving drainage from the inferior petrosal sinus). Although a lateral approach may provide excellent access to the posterolateral jugular foramen, the cranial nerves always remain unexposed deep to the vein. This anatomical configuration is advantageous for removing a paraganglioma because it allows opening of the bulb to resect the tumor while preserving the medial venous wall overlying the lower cranial nerves and is considered the best way to avoid iatrogenic injury. However, when removing lesions originating ventral and medial to the nerves (e.g., chordomas or chondrosarcomas), the robust and often patent jugular vein and cranial nerves may be displaced laterally, challenging an adequate exposure via a transtemporal approach. Third, the entrance of the ICA to the petrous canal and the petrous ICA itself are anterior to the jugular foramen. Anterolateral approaches (e.g., a preauricular subtemporal-infratemporal approach) often require violating the glenoid fossa, temporomandibular joint, and ET, as well as drilling the ICA canal to mobilize the artery anteriorly and gain access to the jugular foramen.

Despite its ability to mitigate some challenges associated with open approaches, the endoscopic endonasal extreme-medial approach to the jugular foramen is not a panacea. In addition to morbidities associated with endoscopic endonasal approaches in general (e.g., cerebrospinal fluid leaks, meningitis, ICA injuries), complications specific to the extreme-medial approach with ET resection may include middle ear effusion with conductive hearing loss that requires a ventilation tube.

In a series of 18 patients who underwent extreme-medial approaches to lesions involving the jugular foramen, $67 \%$ developed ET-related complications, including conductive hearing loss, middle ear effusions, and the need for ventilation tubes. ${ }^{16}$ Of the 15 patients in whom the ET was transected, 4 developed conductive hearing loss. None of the patients in whom the ET was mobilized inferolaterally developed conductive hearing loss. Some of those patients, however, had middle ear effusions.

\section{An Anterolateral Mobilization Strategy for the ET}

Our novel strategy may potentially mitigate some of the morbidity associated with complete ET transection while improving the access afforded by inferolateral mobilization. The new technique anatomically preserves the ET without jeopardizing surgical access to the ventral jugular foramen and hypoglossal canal.

The lateral mobility of the ET is constrained anatomically by 3 components: osseous, fibrocartilaginous, and mucosomuscular elements. The first constraint, the pterygoid process, represents the lateral osseous boundary of the anteromedial segment of the ET. Drilling the medial pterygoid plate does not provide adequate room to transpose the ET laterally. Creating sufficient space to accommodate the laterally mobilized ET is afforded only by drilling the entire pterygoid process. The second constraint is the fibrocartilaginous tissue filling the space between the roof of the ET cartilage and the foramen lacerum and extending posteriorly to attach the ET to the sulcus tubae. The detachment of the ET from the foramen lacerum, the pterygoid tubercle, and all the way posteriorly up to the posterior edge of the medial ovale process is necessary for adequate lateralization. The third constraint is the mucosal attachment of the ET to the floor of the nasopharynx, which can be addressed by dividing this mucosa to mobilize the ET laterally and fully.

To our knowledge, this study is the first to propose a mechanism for anterolateral mobilization of the ET. Previ- 

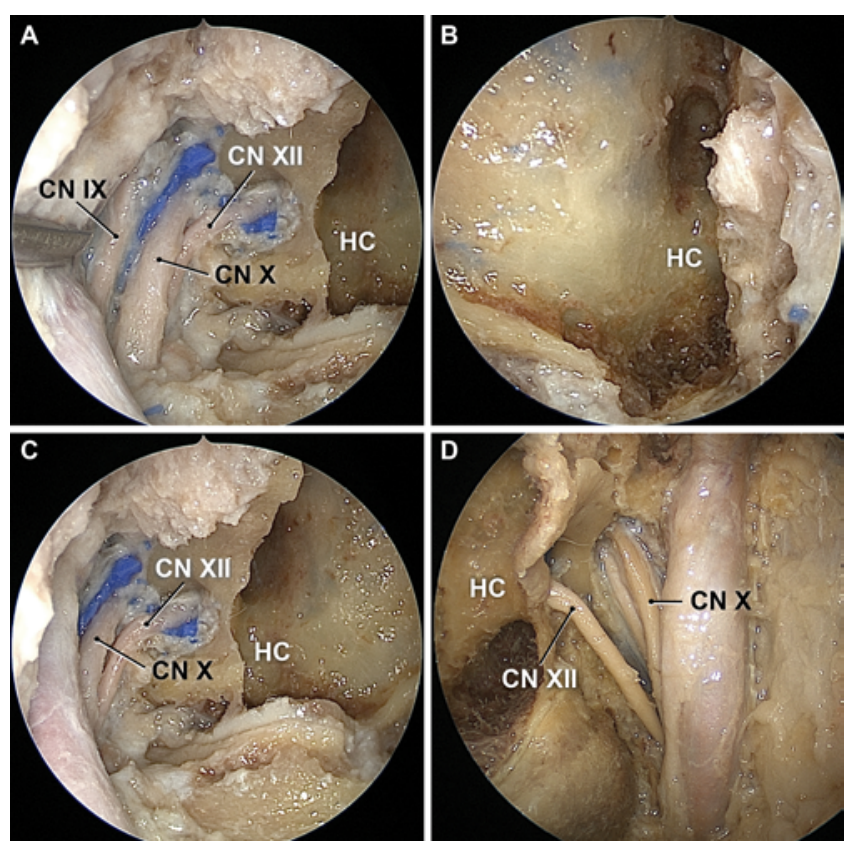

FIG. 5. Qualitative comparison of different ET management strategies for the extreme-medial approach. A: Right anterolateral ET mobilization. B: Left inferolateral ET mobilization. C: Right anterolateral ET mobilization. D: Left complete ET resection. $\mathrm{HC}=$ hypoglossal canal. Panel $\mathrm{D}$ is provided courtesy of Mohamed A. Labib. Copyright Barrow Neurological Institute, Phoenix, Arizona. Published with permission.

ous reports evaluating endoscopic endonasal approaches to the jugular foramen and the poststyloid compartment have addressed the ET either by mobilizing it inferolaterally after its detachment from the fibrocartilaginous tissue overlying the foramen lacerum or by resecting it altogether. ${ }^{1,3,16}$ The anatomical study by Dallan et al. ${ }^{3}$ suggested that fully accessing the jugular foramen requires transection of the ET. Subsequent clinical studies suggested inferolateral mobilization as an alternative in select patients..$^{16}$ To augment the exposure obtained by inferolateral mobilization of the ET, Simal-Julián et al. ${ }^{15}$ used a second trajectory lateral to the ET and medial to the pterygoid muscles. By dissecting the anterior wall of the ET from the pterygoid and tensor veli palatini muscles, they were able to access the stylopharyngeal aponeurosis and the prestyloid compartment. Accessing the retrocarotid area of the poststyloid compartment where the jugular foramen resides was limited, however. ${ }^{15}$

\section{Comparative Anatomical Analysis of ET Management Paradigms}

The ultimate goal of any skull base approach is to obtain adequate surgical exposure to the area of interest with minimal manipulation of neural tissue. Our findings indicate that surgical exposure when approaching the jugular foramen region increases progressively as the surgical strategy escalates from no ET mobilization to partial mobilization, then to complete mobilization, and, finally, to complete ET transection (Figs. 5 and 6). Notably, although the incremental increase in surgical exposure from an intact ET to the anterolateral mobilization of the ET
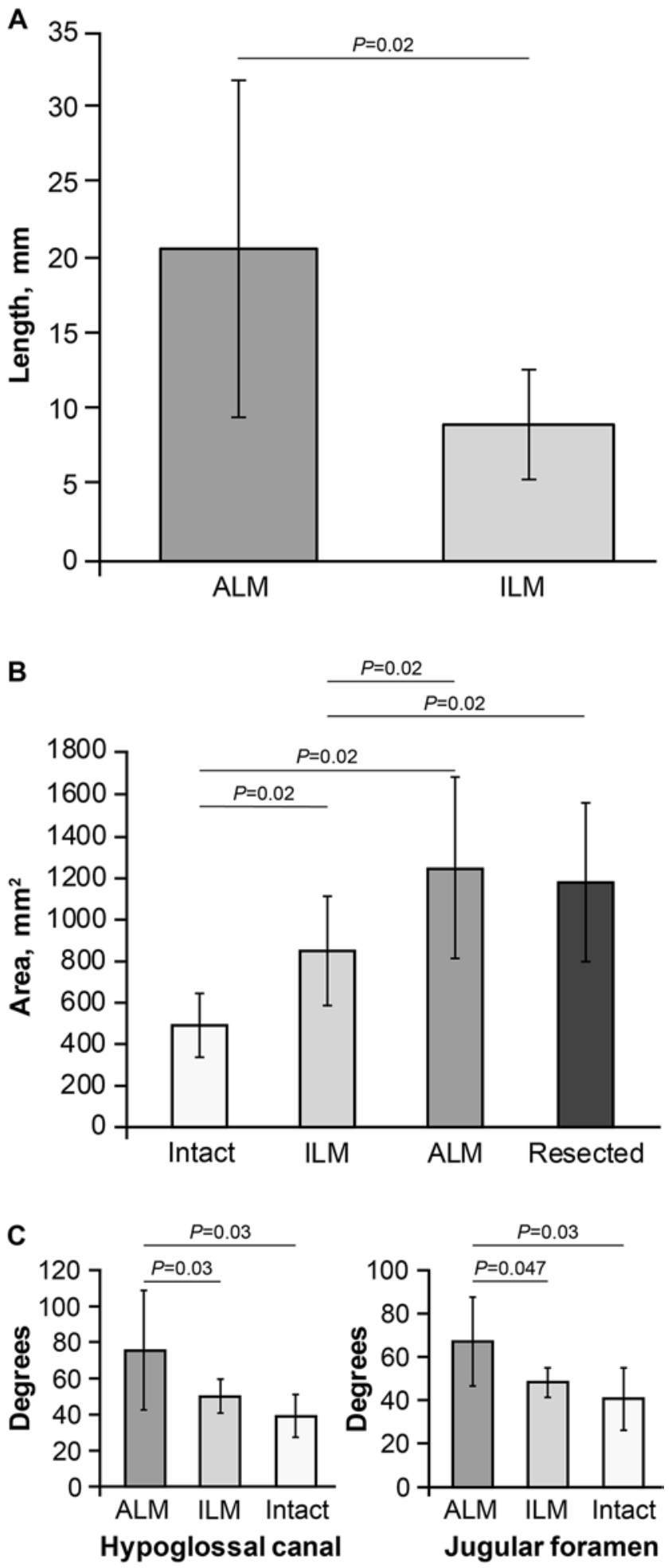

FIG. 6. Results of the quantitative assessment of the approaches $(n=6)$. Comparison of the ET retraction distances $(\mathbf{A})$, surgical exposure area (B), and surgical freedom to the hypoglossal canal and jugular foramen (C) through the endonasal approach with the intact position of the ET, anterolateral mobilization (ALM) of the ET, and inferolateral mobilization (ILM) of the ET. Data are presented as the mean \pm SD (A and C) and as the mean \pm SEM $(B)$. 

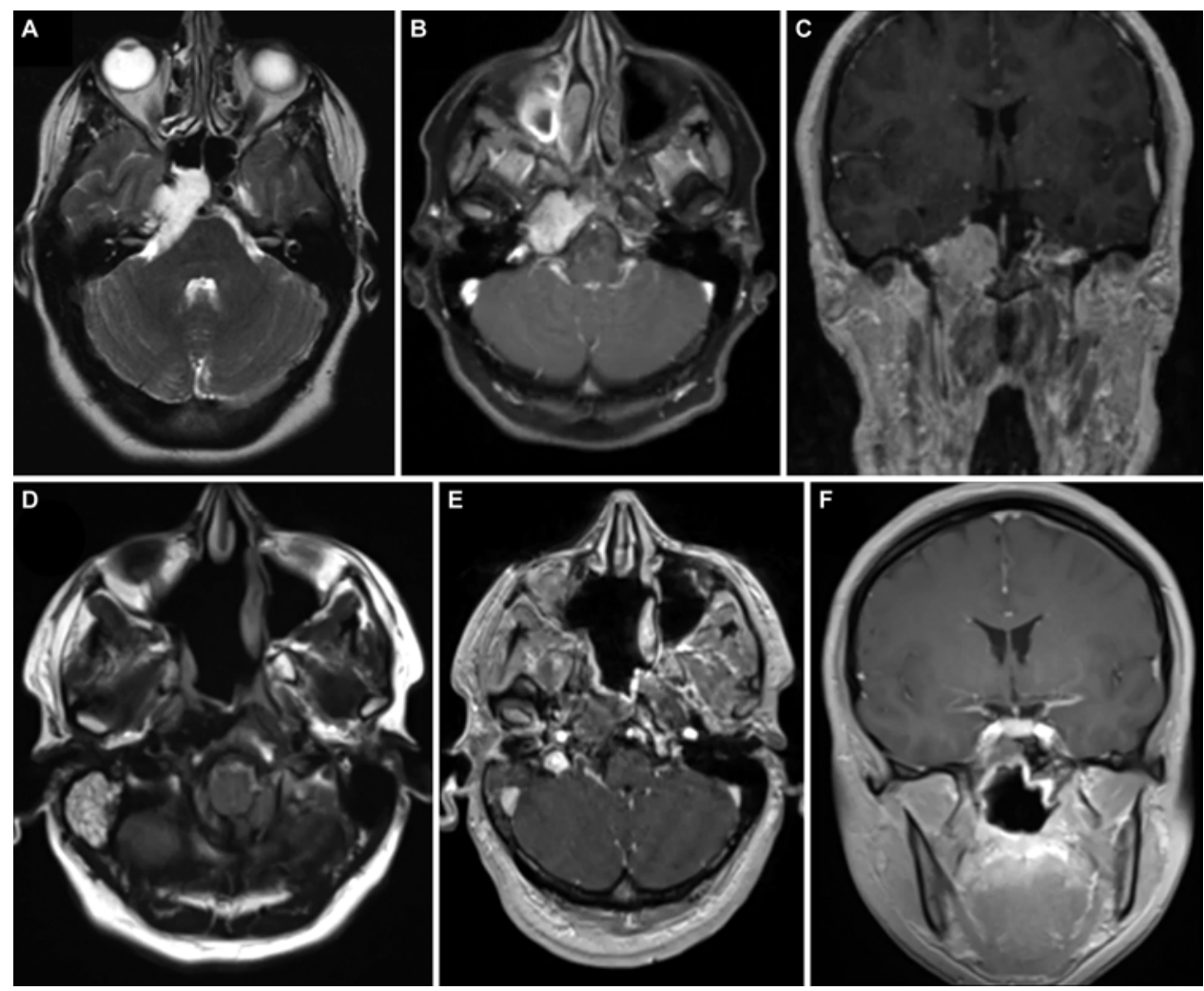

FIG. 7. Case illustration. A: Axial T2-weighted MR image showing a hyperintense lesion involving the right Meckel's cave and petroclival fissure. B: Axial T2-weighted MR image illustrating the caudal extent of the lesion up to the jugular foramen region medial to the parapharyngeal ICA. C: Coronal T1-weighted MR image with contrast showing the rostral-caudal extent of this enhancing tumor. D and E: Axial T1-weighted MR image without (D) and with $(\mathrm{E})$ contrast demonstrating the extent of tumor resection. F: Coronal T1-weighted MR image with contrast confirming gross-total tumor resection.

was statistically significant, no significant difference was observed in exposure obtained with anterolateral mobilization of the ET compared with transecting it altogether. Thus, from a surgical exposure perspective, removing the ET did not improve exposure over that obtained with our anterolateral mobilization technique. In addition, a comparison of the surgical freedom gained by retaining the intact ET compared with inferolateral mobilization or complete lateral mobilization favored anterolateral mobilization.

\section{Technical Considerations}

Performing the anterolateral ET mobilization necessitates full exposure and drilling of the pterygoid process. To be able to accomplish that, the greater palatine canal must be opened, the lesser palatine nerve divided, and the greater palatine nerve lateralized. Sacrificing the lesser palatine nerve will likely cause hemianesthesia of the soft palate. If the greater palatine nerve is inadvertently transected while being lateralized, the hemianesthesia may extend to the ipsilateral hard palate. Hence, we advocate complete unroofing of the grater palatine canal to minimize the risk of inadvertently stretching or transecting the greater palatine nerve.

It is important to emphasize that the additional exposure afforded by the anterolateral mobilization strategy is not at the expense of conventional reconstructive options.
Regardless of the ET management strategy employed, a transpterygoid approach with scarification of the ipsilateral sphenopalatine artery is an essential step to obtain adequate access to the petroclival region. Hence, sphenopalatine artery-based flaps must be harvested on the contralateral side of the extreme medial approach. In the absence of a viable contralateral nasoseptal flap, other options, including a lateral vault flap or a temporoparietal fascial flap, may be considered.

\section{Study Limitations and Future Goals}

This study has several limitations that will be addressed in the future. First, the concept of anterolateral ET mobilization is based on anatomical relationships evaluated and analyzed in cadaveric specimens. While the anatomical points selected to measure areas of exposure and surgical freedom were identifiable in and comparable among the different study groups, those points remain artificial in the clinical setting, because they may be destroyed by aggressive lesions. Furthermore, dynamic endoscopy and angled lenses may facilitate the exposure in some situations without needing to mobilize the ET in any way. To be completely validated, the anterolateral ET mobilization strategy must be performed in the operating room. Second, although the proposed ET mobilization technique is intended to preserve the ET anatomically, functional conservation of the ET can be proved only in clinical studies. 
Nonetheless, the present study shows that the degree of surgical freedom and extent of exposure attained by ET resection and anterolateral mobilization are comparable. Accordingly, every effort must be made to mobilize the ET anterolaterally instead of resecting it. The case discussed above would have been ideal for treatment with anterolateral mobilization instead of resection. Finally, the exposure advantage obtained by anterolaterally mobilizing the ET instead of resecting it must be weighed against the potential increase in operative time that may be associated with the new technique. Although the measurement of any additional operative time can only be performed in future clinical settings, we believe that the disadvantage of prolonging the surgery may be offset by the potential advantage of minimizing the risks associated with completely resecting the ET.

\section{Conclusions}

The ventral aspect of the jugular foramen and infrapetrous region are among the most difficult to access surgically. While offering a potential alternative to the more conventional lateral and posterolateral approaches to this region, the endoscopic endonasal extreme-medial approach is vastly obstructed by the anatomical relationships of the ET. Transecting the ET to maximize access has been associated with various complications. In this study, a novel strategy to mobilize the ET anterolaterally was introduced and was shown to be superior to other ET mobilization techniques while providing comparable access and degree of surgical freedom to ET resection. This work provides a sound anatomical foundation for the surgical strategy. Clinical application and outcomes remain to be demonstrated in future studies.

\section{Acknowledgments}

We are grateful for financial support from the Newsome Chair in Neurosurgery Research held by Dr. Preul and the Barrow Neurological Foundation.

We thank the staff of Neuroscience Publications at Barrow Neurological Institute for assistance with manuscript and video preparation.

\section{References}

1. Al-Sheibani S, Zanation AM, Carrau RL, Prevedello DM, Prokopakis EP, McLaughlin N, et al: Endoscopic endonasal transpterygoid nasopharyngectomy. Laryngoscope 121:2081-2089, 2011

2. Brackmann DE: The facial nerve in the infratemporal approach. Otolaryngol Head Neck Surg 97:15-17, 1987

3. Dallan I, Bignami M, Battaglia P, Castelnuovo P, Tschabitscher M: Fully endoscopic transnasal approach to the jugular foramen: anatomic study and clinical considerations. Neurosurgery 67 (3 Suppl Operative):ons1-ons8, 2010

4. Fisch U, Fagan P, Valavanis A: The infratemporal fossa approach for the lateral skull base. Otolaryngol Clin North Am 17:513-552, 1984

5. Goldenberg RA, Gardner G: Tumors of the jugular foramen: surgical preservation of neural function. Otolaryngol Head Neck Surg 104:129, 1991

6. Hakuba A, Hashi K, Fujitani K, Ikuno H, Nakamura T, Inoue Y: Jugular foramen neurinomas. Surg Neurol 11:83-94, 1979

7. Jackson CG, Cueva RA, Thedinger BA, Glasscock ME III:
Cranial nerve preservation in lesions of the jugular fossa. Otolaryngol Head Neck Surg 105:687-693, 1991

8. Kassam A, Snyderman CH, Mintz A, Gardner P, Carrau RL: Expanded endonasal approach: the rostrocaudal axis. Part I. Crista galli to the sella turcica. Neurosurg Focus 19(1):E3, 2005

9. Kassam A, Snyderman CH, Mintz A, Gardner P, Carrau RL: Expanded endonasal approach: the rostrocaudal axis. Part II. Posterior clinoids to the foramen magnum. Neurosurg Focus 19(1):E4, 2005

10. Lee DL, McCoul ED, Anand VK, Schwartz TH: Endoscopic endonasal access to the jugular foramen: defining the surgical approach. J Neurol Surg B Skull Base 73:342-351, 2012

11. Morera VA, Fernandez-Miranda JC, Prevedello DM, Madhok R, Barges-Coll J, Gardner P, et al: "Far-medial" expanded endonasal approach to the inferior third of the clivus: the transcondylar and transjugular tubercle approaches. Neurosurgery 66 (6 Suppl Operative):211-220, 2010

12. Patel SJ, Sekhar LN, Cass SP, Hirsch BE: Combined approaches for resection of extensive glomus jugulare tumors. A review of 12 cases. J Neurosurg 80:1026-1038, 1994

13. Rhoton AL Jr: Jugular foramen. Neurosurgery 47 (3 Suppl):S267-S285, 2000

14. Samii M, Babu RP, Tatagiba M, Sepehrnia A: Surgical treatment of jugular foramen schwannomas. J Neurosurg 82:924-932, 1995

15. Simal-Julián JA, Miranda-Lloret P, Beltrán-Giner A, PlazaRamirez E, Botella-Asunción C: Full endoscopic endonasal extreme far-medial approach: Eustachian tube transposition. J Neurosurg Pediatr 11:584-590, 2013

16. Vaz-Guimaraes F, Nakassa ACI, Gardner PA, Wang EW, Snyderman CH, Fernandez-Miranda JC: Endoscopic endonasal approach to the ventral jugular foramen: anatomical basis, technical considerations, and clinical series. Oper Neurosurg (Hagerstown) 13:482-491, 2017

\section{Disclosures}

Dr. Nakaji: stock ownership in GT Medical Technologies and royalties from OsteoMed. Dr. Carrau: ad hoc consultant for Medtronic, PLC, and Stryker Corp.; instrument royalties from KLS Martin Group. Dr. Prevedello: consultant for Stryker, Integra, and Medtronic; honoraria and royalties from Mizuho; royalties from KLS Martin Group, and other research support from Integra and Storz. Dr. Little: ownership stake in Kogent; consultant for SPIWay. Dr. Youssef: direct stock ownership in GT Medical Technologies; royalties from Mizuho.

\section{Author Contributions}

Conception and design: Labib, Belykh. Acquisition of data: Labib, Cavallo, Zhao, Ferreira. Analysis and interpretation of data: Labib, Belykh. Drafting the article: Labib. Critically revising the article: Nakaji, Prevedello, Carrau, Little, Youssef. Reviewed submitted version of manuscript: all authors. Approved the final version of the manuscript on behalf of all authors: Nakaji. Statistical analysis: Zhao. Administrative/technical/material support: Preul. Study supervision: Nakaji, Preul.

\section{Supplemental Information Videos}

Video 1. https://vimeo.com/382399298.

\section{Correspondence}

Peter Nakaji: c/o Neuroscience Publications, Barrow Neurological Institute, St. Joseph's Hospital and Medical Center, Phoenix, AZ. neuropub@barrowneuro.org. 